\title{
Efficient Robust Digital Annulus Fitting with Bounded Error*
}

\author{
Minh Son Phan ${ }^{1,2}$, Yukiko Kenmochi ${ }^{1}$, Akihiro Sugimoto ${ }^{3}$, Hugues Talbot ${ }^{1}$, \\ Eric Andres ${ }^{4}$, and Rita Zrour ${ }^{4}$ \\ 1 Université Paris-Est, LIGM, UPEMLV-ESIEE-CNRS, France \\ 2 Université de Strasbourg, LSIIT, France \\ 3 National Institute of Informatics, Japan \\ ${ }^{4}$ Laboratory XLIM, SIC Department, University of Poitiers, France
}

\begin{abstract}
A digital annulus is defined as a set of grid points lying between two circles sharing an identical center and separated by a given width. This paper deals with the problem of fitting a digital annulus to a given set of points in a $2 \mathrm{D}$ bounded grid. More precisely, we tackle the problem of finding a digital annulus that contains the largest number of inliers. As the current best algorithm for exact optimal fitting has a computational complexity in $O\left(N^{3} \log N\right)$ where $N$ is the number of grid points, we present an approximation method featuring linear time complexity and bounded error in annulus width, by extending the approximation method previously proposed for digital hyperplane fitting. Experiments show some results and runtime in practice.
\end{abstract}

Keywords: fitting, annulus, approximation, halfspace range searching.

\section{Introduction}

Shape fitting is widely used in computer vision, computational geometry, image analysis and many other areas. Given a set of points $S$, a shape model $M$ (for example, lines, curves, planes, or circles), and some criterion $F$, the problem is to fit $M$ to $S$ under $F$. Depending on the criteria employed, we have several well-known methods dealing with this problem. The method of least-squares [1] minimizes the sum of squared residuals from all given points. This method is popular, but it is not efficient in the presence of outliers. In order to enhance the robustness, many alternative methods have also been proposed, such as the method of least-absolute-values [2], the method of least-median-of-squares [3]. However, these methods are still not robust in the presence of many outliers. One of the commonly used methods for robust shape fitting is RANdom SAmple Consensus (RANSAC) 4]. It can provide robust results even if there is a significant number of outliers, with reasonable runtime in practice, while it provides neither guarantee of optimal solution nor bound of approximation error.

* This work has been partly supported by the French Agence Nationale de la Recherche (ANR-2010-BLAN-0205 03) and a French-Japanese joint research project called the Sakura program (No. 27608XJ).

R. Gonzalez-Diaz, M.-J. Jimenez, B. Medrano (Eds.): DGCI 2013, LNCS 7749, pp. 253-264, 2013.

(C) Springer-Verlag Berlin Heidelberg 2013 
In this paper, we use a criterion similar to that of RANSAC. As we assume that our input data is discrete, such as a digital image, we use a digital shape model, instead of a continuous one as generally used for the above mentioned conventional methods. Fitting of digital lines [5] or digital planes [6] has been already studied. This paper aims at exploring the problem of fitting a digital circle. We consider a digital circle as a digital annulus [7, defined as a set of grid points lying between two circles sharing a center but with different radii. This paper treats the problem of fitting a digital annulus to a given set of points in a 2D bounded grid. More precisely, we tackle the problem of finding a digital annulus that contains the largest number of inliers. The current best algorithm for exact optimal fitting has a computational complexity in $O\left(N^{3} \log N\right)[8$. We present an approximation method featuring linear time complexity and bounded error in annulus width by extending an approximation method proposed for digital hyperplane fitting [6].

We first formulate our digital annulus fitting as the digital plane fitting problem with some particular setting. For our approximation method, we then employ the approximate halfspace range searching technique 9], which consists of two main steps: generating an approximate range space, and providing a data structure to solve approximate halfspace range searching with a constant query time. We follow these two steps, in the context of solving our converted digital plane fitting, by combining two problems of approximate halfspace range searching. We also explain how to interpret results of digital plane fitting in the context of digital annulus fitting, with an interpretation of bounded error. Finally, we provide experimental results and measured runtimes.

\section{Problem of Digital Annulus Fitting}

\subsection{Digital Annulus and Its Fitting Problem}

In this paper, we follow the definition of a digital annulus as introduced by Andres et al. [7] and used by Zrour et al. for fitting [8]. We first describe the continuous model of circles. A continuous circle $\mathbf{C}$ with center $(a, b)$ and radius $r$ in the Euclidean space $\mathbb{R}^{2}$ is defined by

$$
\mathbf{C}=\left\{(x, y) \in \mathbb{R}^{2}:(x-a)^{2}+(y-b)^{2}=r^{2}\right\}
$$

where $a, b, r \in \mathbb{R}$. In the discrete space $\mathbb{Z}^{2}$, the digitization of (1) is defined by a set of grid points lying between two circles that share the same center $(a, b)$ and two different radii $r-\frac{w}{2}$ and $r+\frac{w}{2}$, where $w \in \mathbb{R}$ is the width between the two circles. Namely,

$$
\mathbf{A}=\left\{(x, y) \in \mathbb{Z}^{2}:\left(r-\frac{w}{2}\right)^{2} \leq(x-a)^{2}+(y-b)^{2} \leq\left(r+\frac{w}{2}\right)^{2}\right\}
$$

This is called a digital annulus, or Andres circle [7. Given a set $S$ of discrete points coming from a $[0, \delta]^{2}$ grid, where $\delta \in \mathbb{N}$, the problem is to fit this digital 
annulus model A to $S$ such that the fitted digital annulus contains as many points of $S$ as possible, called inliers. This problem is described below. Points that are not contained in the model are called outliers.

\section{Problem 1 (Digital annulus fitting)}

Input : $A$ set $S$ of discrete points bounded in a $[0, \delta]^{2}$ grid, and a fixed width $w$. Output: A digital annulus A containing the maximum number of inliers in $S$.

This digital annulus fitting problem is solved by an exact method, introduced in [8], in time $O\left(|S|^{3} \log |S|\right)$. This complexity of the exact solution is not feasible in practice when $|S|$ is large. In some cases it may be sufficient to compute an approximate solution if the solution is obtained with inexpensive computational complexity. We thus propose here an approximate method with linear runtime and bounded error, instead of searching for the exact solution. For this purpose, we first convert our digital annulus fitting problem into a more simple problem in the following.

\subsection{Digital Plane Fitting Induced by Digital Annulus Fitting}

Since the inequalities (2) are non-linear with respect to the unknown parameters $a, b, r$, we convert those to linear ones along the known conversion [10]. Then, the digital annulus formula of (2) can be converted into the digital plane formula [11, defined by two inequalities of the form $0 \leq A x+B y+z+C \leq W$ where $W$ is the width.

The inequalities (2) are written as:

$$
\left(r-\frac{w}{2}\right)^{2} \leq-2 a x-2 b y+x^{2}+y^{2}+a^{2}+b^{2} \leq\left(r+\frac{w}{2}\right)^{2} .
$$

Letting

$$
\left\{\begin{array}{l}
z=x^{2}+y^{2} \\
A=-2 a \\
B=-2 b \\
C=a^{2}+b^{2}-\left(r-\frac{w}{2}\right)^{2} \\
C^{\prime}=a^{2}+b^{2}-\left(r+\frac{w}{2}\right)^{2}
\end{array}\right.
$$

we have that

$$
0 \leq A x+B y+z+C \leq C-C^{\prime}
$$

We can thus define the converted digital plane model as follows:

$$
\mathbf{P}=\left\{(x, y, z) \in \mathbb{Z}^{3}: 0 \leq A x+B y+z+C \leq C-C^{\prime}\right\} .
$$

Obviously this has the form of a digital plane [11, which is defined as a set of grid points lying between two parallel planes, whose width is set to be $W=C-C^{\prime}$. Therefore, (5) leads to the following observation.

Observation 1. The problem of digital annulus fitting is equivalent to the problem of digital plane fitting for the model defined by (5)).

This converted problem is described as follows. 


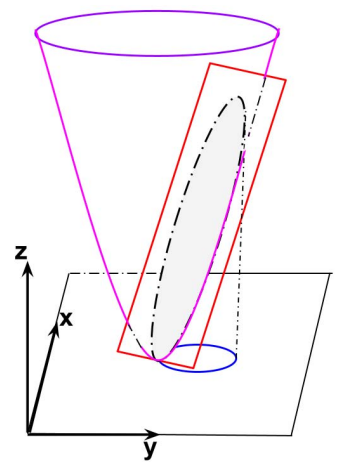

Fig. 1. Illustration of converting a circle (colored in blue) in $2 \mathrm{D}$ into a plane (colored in red) in $3 \mathrm{D}$. The converted $3 \mathrm{D}$ points are on the parabola $z=x^{2}+y^{2}$ (colored in violet).

\section{Problem 2 (Converted digital plane fitting)}

Input : A set $S$ of discrete points bounded in a $[0, \delta]^{2}$ grid, and width $w$. Output: A digital plane $\mathbf{P}$ containing the maximum number of inliers when the input set is $S^{\prime}=\left\{\left(x, y, x^{2}+y^{2}\right):(x, y) \in S\right\}$.

Figure1illustrates the relationship between $\mathbf{A}$ and $\mathbf{P}$, so that the 2D coordinates $(x, y)$ of $\mathbf{A}$ are converted into 3D coordinates $\left(x, y, x^{2}+y^{2}\right)$ of $\mathbf{P}$. In fact, the setting of new parameters in (4) is not efficient since $\forall(x, y) \in[0, \delta]^{2} \Rightarrow z=$ $x^{2}+y^{2} \in\left[0,2 \delta^{2}\right]$, and consequently the $2 \mathrm{D}$ grid of size $[0, \delta]^{2}$ for digital annulus fitting is changed to the $3 \mathrm{D}$ grid of size $[0, \delta]^{2} \times\left[0,2 \delta^{2}\right]$ for the converted digital plane fitting; the grid size for $z$ is too large in practice. We therefore define, instead of (4),

$$
\left\{\begin{array}{l}
z=\frac{1}{2 \delta}\left(x^{2}+y^{2}\right), \\
A=-\frac{a}{\delta}, \\
B=-\frac{b}{\delta}, \\
C=\frac{1}{2 \delta}\left(a^{2}+b^{2}-\left(r-\frac{w}{2}\right)^{2}\right), \\
C^{\prime}=\frac{1}{2 \delta}\left(a^{2}+b^{2}-\left(r+\frac{w}{2}\right)^{2}\right),
\end{array}\right.
$$

by dividing all the terms in (3) by $2 \delta$. With this setting, we see that $x, y \in$ $[0, \delta] \Rightarrow z=\frac{1}{2 \delta}\left(x^{2}+y^{2}\right) \in[0, \delta]$, so that we have a $3 \mathrm{D}$ grid $[0, \delta]^{3}$ for digital plane fitting.

Concerning the ranges of the new parameters $A, B, C$ and $C^{\prime}$, we first obtain $a, b \in[0, \delta] \Rightarrow A=-\frac{a}{\delta}, B=-\frac{b}{\delta} \in[-1,0]$. Accordingly, the search space for the converted digital plane is reduced, compared with the standard digital plane fitting. To enforce $\left(r-\frac{w}{2}\right)^{2}$ and $\left(r+\frac{w}{2}\right)^{2}$ to be positive, $C$ and $C^{\prime}$ in (6) must satisfy

$$
C, C^{\prime} \leq \frac{a^{2}+b^{2}}{2 \delta} \text { or equivalently, } C, C^{\prime} \leq \frac{\delta\left(A^{2}+B^{2}\right)}{2} .
$$


This indicates that the ranges of the parameters $C$ and $C^{\prime}$ depend on those of $A$ and $B$, and we can simply specify that $C, C^{\prime} \in\left[-\delta, \frac{\delta\left(A^{2}+B^{2}\right)}{2}\right]$.

We also recall that the width $W$ of a digital plane, converted from a digital annulus, is given by

$$
W=C-C^{\prime}=\frac{r w}{\delta}
$$

after the normalization of (6). This indicates that $W$ is not constant, but depends on the radius $r$ of the digital annulus. Therefore, our converted digital plane fitting problem is different from the standard one [6] in that the width $W$ is not a constant. Since we obtain from (6)

$$
r=\sqrt{\frac{\delta\left(A^{2}+B^{2}\right)}{2}-2 \delta C}+\frac{w}{2},
$$

we can also say that $W$ depends on $A, B$ and $C$. In other words, once we set the values for $A, B$ and $C$, we can automatically set the value for $r$, and thus for $W$ as well.

For this converted digital plane fitting problem, we apply the approximate method, which is originally proposed for the standard digital plane fitting problem [6. We show in the next section that the dependency of $W$ on $A, B$ and $C$ does not critically obstruct applying the approximate method to our converted digital plane fitting problem.

\section{Approximate Method for Digital Annulus Fitting}

\subsection{Method Outline}

The proposed digital annulus fitting algorithm consists of four steps, as described in Algorithm 1. In the first step, we convert the problem of digital annulus fitting into a problem of digital plane fitting by using the notions presented in the previous section. In other words, a set of points $S$ in the $2 \mathrm{D}$ grid of size $[0, \delta]^{2}$ is transformed into the set of points $S^{\prime}=\left\{\left(x, y, \frac{x^{2}+y^{2}}{2 \delta}\right):(x, y) \in S\right\}$ in the 3D grid of size $[0, \delta]^{3}$. In the second step, we build a data structure with respect to $S^{\prime}$ for the approximate halfspace range searching algorithm. More precisely, we specify a query set $\mathbb{H}$ for approximate halfspace range searching with a given bounded error $\varepsilon$, and generate a data structure that allows to answer the following query: for any plane $h \in \mathbb{H}$, how many points of $S^{\prime}$ are on or below $h$ with the bounded error $\varepsilon$. In the third step, we first modify the query for the digital plane fitting with width $W+5 \varepsilon$, i.e., we count the number of points of $S^{\prime}$ that lie between two parallel planes with distance $W+5 \varepsilon$. We then find an approximate digital plane with the maximum number of inliers. The final step is to interpret the result of the converted problem as that of the original digital annulus problem.

As Steps 1 and 4 are already explained in the previous section, we describe Steps 2 and 3. Furthermore, we discuss the complexity of our proposed method. 


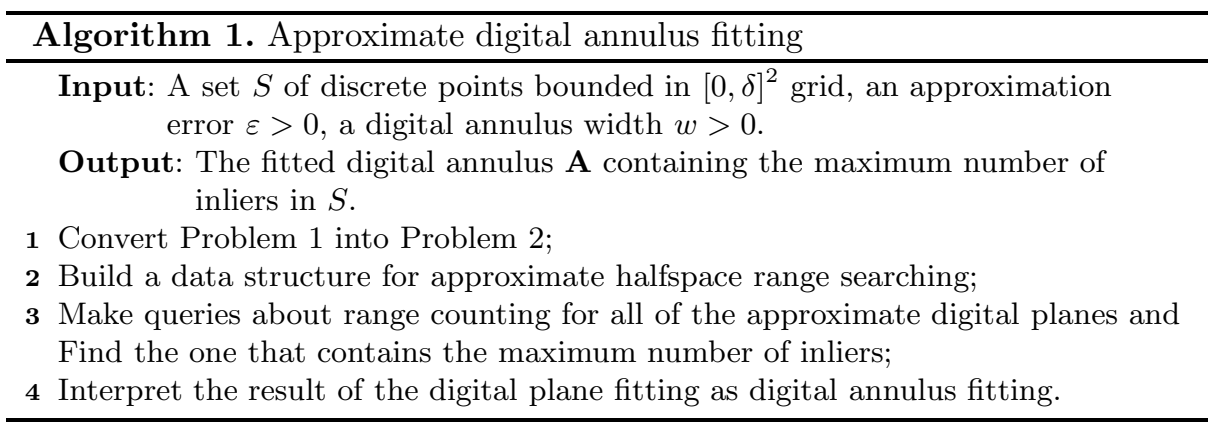

\subsection{Data Structure for Approximate Halfspace Range Searching}

As this part is based on the work of Fonseca et al. 9], we first summarize their method, and then explain the changes suitable for our problem.

Given a set of $3 \mathrm{D}$ points $T$ and a plane $h$ in the form $A x+B y+z+C=0$, halfspace range searching (or counting) is the problem of counting the number of points of $T$ that are on, or below $h$. In order to solve this problem, Fonseca et al. [9] have proposed an approximate method with a bounded error $\varepsilon>0$.

One of the main ideas of the method is to define a sufficiently large finite set of planes $\mathbb{H}$, so that any query plane is approximated by some plane in $\mathbb{H}$ with bounded error $\varepsilon$. In this paper, $\mathbb{H}$ is obtained by generating different slopes $A, B \in[-1,0]$ and different intercepts $C \in\left[-\delta, \frac{\delta\left(A^{2}+B^{2}\right)}{2}\right]$; for their ranges, see the previous section. For $A$ and $B$, the interval of their variations should be set to be $\frac{\varepsilon}{\delta}$, while it should be $\varepsilon$ for $C$ (see [9] for more details).

For each plane $h \in \mathbb{H}$, we compute a counting function $f(T \cap h)$ that returns approximately the number of points of $T$ that are on, or below $h$. The objective of constructing a data structure is to compute this function $f(T \cap h)$ for all the planes $h \in \mathbb{H}$. Points within distance $\varepsilon$ from a plane $h$ may or may not be reported.

The computational complexity is described as follows:

Theorem 1. (Fonseca et al. [9]) For a set of $N$ points in the $[0,1]^{3}$ unit grid and some $\varepsilon>0$, one can build a data structure with $O\left(\varepsilon^{-3}\right)$ storage space, in $O\left(N+\varepsilon^{-3} \log ^{O(1)}\left(\varepsilon^{-1}\right)\right)$ time, such that for a given query plane $h$, the number of points on or below $h$ can be approximately reported in $O(1)$ time, in the following sense: all the points (below $h$ ) that have a larger distance than $\varepsilon$ from $h$ are counted. Points that are closer to $h$ on both sides may or may not be reported.

In fact, in order to obtain this complexity (linear to $N$ ), we build a data structure based on an octree. Let us call a $[0, \delta]^{3}$ grid a primitive cube, which is divided into 8 children. Its children are also divided recursively into 8 subcubes, until the size of divided cubes equals $\varepsilon$. Let $X$ be a cube generated from a primitive cube, and $T$ be a set of points bounded by $X$ in dimension $\delta$. We then verify whether each plane of $\mathbb{H}$ passes through $X$, and compute $f(T \cap h), \forall h \in \mathbb{H}$ in the following way. Let $X_{i}$ denote the children of $X$, for $i=1 . .8$, and $T_{i}=X_{i} \cap T$. 
We then compute $f(T \cap h)$ by summarizing all the results from its children $f\left(T_{i} \cap h\right)$, which is also recursively computed, as follows:

$$
f(T \cap h)= \begin{cases}\sum_{i=1}^{8} f\left(T_{i} \cap h\right) \text { if the size of } X \text { is larger than } \varepsilon \text { and } h \cap X \neq \emptyset, \\ 0 & \text { if the size of } X \text { is larger than } \varepsilon \text { and } h \text { is below } X, \\ |T| & \text { otherwise. }\end{cases}
$$

Finally, the data structure of a primitive cube is built recursively. Figure 2 illustrates a $2 \mathrm{D}$ example for computing the counting function $f(T \cap h)$ of (9).

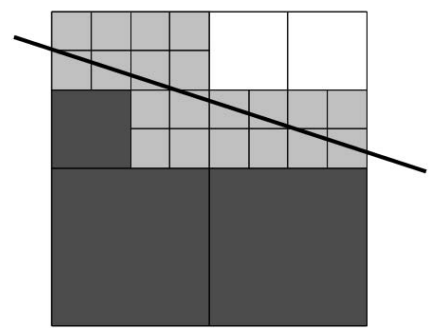

Fig. 2. Illustration for recursively computing $f(T \cap h)$ in a $2 \mathrm{D}$ case (a line) by dividing a primitive square (corresponding to a cube in $3 \mathrm{D}$ ) into 4 children. The light grey cells are the squares of size $\varepsilon$ through which a query line $h$ goes, while the dark grey cells are the squares below $h$. See (9) for the definition.

\subsection{Query for Approximate Digital Plane Fitting}

After building a data structure for a set of points $S^{\prime}$ in the $[0, \delta]^{3}$ grid, we count the number of points lying in every digital plane, i.e., between a pair of parallel planes with distance $W$. In order to calculate it approximately, we need a finite query set $\mathbb{Q}$ of approximate digital planes for our converted digital plane fitting as follows. For each plane $h \in \mathbb{H}$, we take the values of $A, B$ and $C$ of $h$ for the digital plane parameters $A, B$, and $C$, respectively. Once the values of $A, B$ and $C$ are fixed, we can automatically set $C^{\prime}$ or $W$ from (7) and (8), i.e., the rest of the digital plane parameters. To generate a query for approximate digital plane fitting with a width $W$, we refer to the following theorem presented in [6] 1$]$

Theorem 2. (Aiger et al. [6]) Given a set of $N$ points in $[0, \delta]^{3}$, and some $\varepsilon>0, W>0$, a digital plane of width $W+5 \varepsilon$ that contains $n>n_{\text {opt }}$ points, can be found in $O\left(N+\left(\frac{\delta}{\varepsilon}\right)^{3} \log ^{O(1)}\left(\frac{\delta}{\varepsilon}\right)\right)$ time, where $n_{\text {opt }}$ is the maximum number of points that any digital plane of width $W$ in $[0, \delta]^{3}$ can contain.

${ }^{1}$ Even if the original theorem was proposed for $N$ points on a $[0, \delta]^{3}$ grid, the theorem itself, as well as the proposed method, is established for a more general setting such that the $N$ points can simply be in $[0, \delta]^{3}$ regardless of any grid. 
In fact, we need to generate a new query set $\mathbb{Q}$ such that any digital plane with width $W$ is completely contained in at least one of the digital planes in $\mathbb{Q}$. Theorem 2 indicates that such query digital planes must have a width of at least $W+5 \varepsilon$. In other words, due to this setting, the important property, $n \geq n_{\text {opt }}$, is guaranteed. Even if $W$ is not constant as in this paper, because it depends on each parameter set of $A, B$ and $C$, this result is still valid.

An approximate solution of the converted digital plane fitting is obtained by finding the digital plane in $\mathbb{Q}$ in which the number of points is maximized.

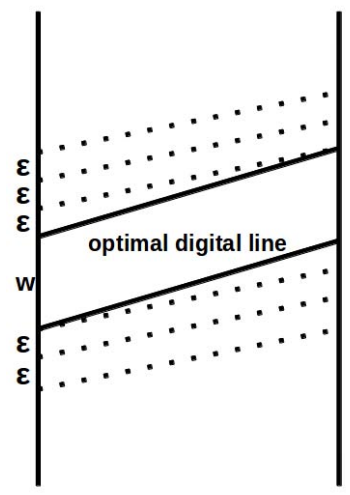

Fig. 3. Relationship between approximate (dotted line) and optimal digital line (continuous line). There is at least one approximate digital line that contains the optimal digital line.

\subsection{Complexity of the Approximate Digital Annulus Fitting}

As a result, we obtain the next corollary from Theorem 2 after minor adaptations.

Corollary 1. Given a set of $N$ points on a grid $[0, \delta]^{2}$, and some $\varepsilon>0, w>0$, a digital annulus of width $w+5 \frac{\delta}{r} \varepsilon$ that contains $n>n_{\text {opt }}$ points, can be found in $O\left(N+\left(\frac{\delta}{\varepsilon}\right)^{3} \log ^{O(1)}\left(\frac{\delta}{\varepsilon}\right)\right)$ time, where $r$ is the radius of a digital annulus of width $w+5 \frac{\delta}{r} \varepsilon$ and $n_{\text {opt }}$ is the maximum number of points that any digital annulus of width $w$ in $[0, \delta]^{2}$ can contain.

This corollary shows that the proposed method has linear computational complexity with respect to $N$. Obviously, if we decrease the bounded error $\varepsilon$, the computational time increases due to the second term, just like for approximated digital plane fitting; see Theorem 2 . The difference to Theorem 2 is that $\varepsilon$ cannot be tuned directly in the original $2 \mathrm{D}$ grid for digital annulus fitting, but in the $3 \mathrm{D}$ grid for the converted digital plane fitting. In other words, we can give $\varepsilon$ as a constant ambiguous zone around the border of a digital plane in 3D (i.e. a zone in which points may or may not be counted), but once we project it to 


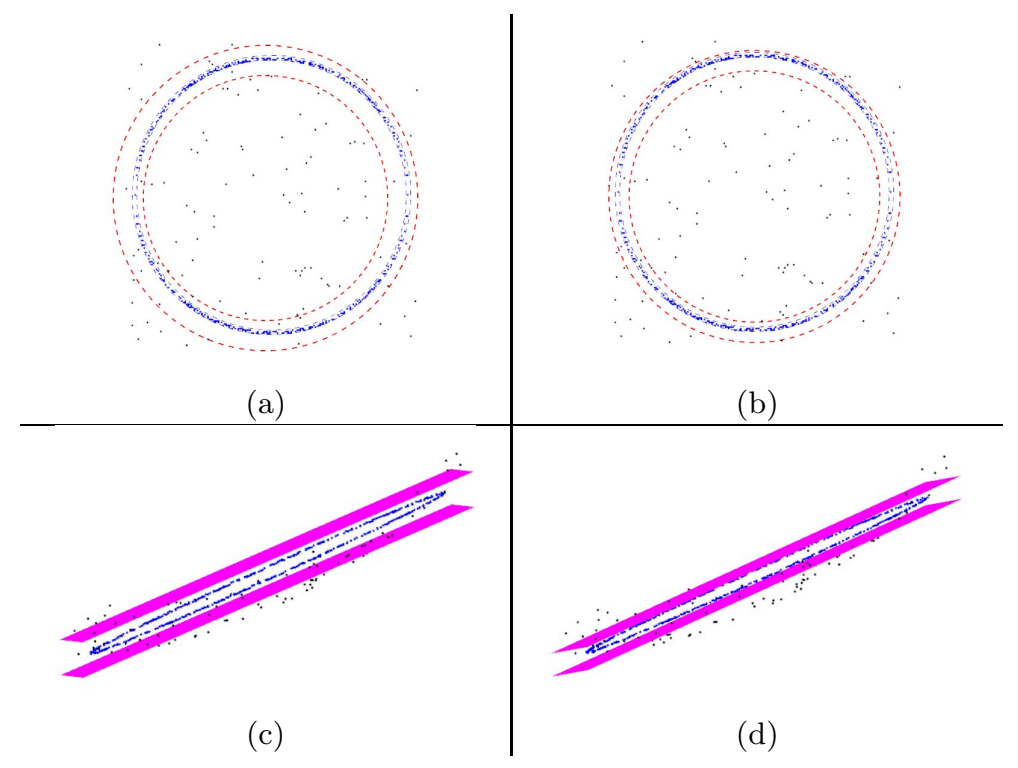

Fig. 4. The fitted digital annuli (colored in red) for $\varepsilon=1.5$ (a) and $\varepsilon=1.0$ (b). They contain the true digital annulus (colored in blue). The converted digital planes are also illustrated for $\varepsilon=1.5$ (c) and $\varepsilon=1.0(\mathrm{~d})$.

$2 \mathrm{D}$ before the conversion, the corresponding ambiguous zone becomes $\frac{\delta}{r} \varepsilon$ which is obtained from (7).

\section{Experiments}

The 2D point cloud data used for the experiments were created as follows. By using the digital annulus $\mathbf{A}$ of (2) with $w=3.0, a=b=100.0$, we randomly generated 400 inliers that satisfy $\mathbf{A}$, and $100 n$ outliers that do not satisfy $\mathbf{A}$, for $n=0, \ldots, 10$; there are 11 variations for the number of outliers. All of the generated points are bounded in the $[0,200]^{2}$ grid. The values for the parameters of the converted digital planes $\mathbf{P}$ of (5) are also computed: $A=B=-0.5$, $C=30.1975$ and $C^{\prime}=28.84$. Note that the experiments were run on a standard $\mathrm{PC}$ with core $\mathrm{i} 3 \mathrm{CPU}$ at $2.20 \mathrm{GHz}$.

In the first experiment, we had $n=1$ constant (i.e., 100 outliers) for the input data and observed the fitting results by using different bounded errors such that $\varepsilon=3.0,2.5,2.0,1.5,1.0$. As seen in Table 1 and Figure 4 the number of inliers decreases as $\varepsilon$ becomes smaller, and it tends to converge to the ground-truth solution. Clearly, the smaller the value of $\varepsilon$, the more precise the solution. Concerning the runtime, it is in fact polynomial in factor $\varepsilon$ (see Figure 6). Therefore, we need to select an appropriate $\varepsilon$ (for example 1.5 or 1.0 for this experiment) 
Table 1. Results of digital annulus fitting with varied $\varepsilon$ to the data with $n=1$

\begin{tabular}{|c|c|c|c|c|c|c|c|c|c|}
\hline$\varepsilon$ & runtime & \multicolumn{7}{|c|}{ parameters } \\
\cline { 3 - 10 } & $\begin{array}{c}\text { approx } \\
\text { method })\end{array}$ & $a$ & $b$ & $r$ & $A$ & $B$ & $C$ & $C^{\prime}$ & inliers \\
\hline 3.0 & $7.80 \mathrm{sec}$ & 96.0 & 96.0 & 89.62 & -0.48 & -0.48 & 34.0 & 18.0 & 441 \\
\hline 2.5 & $13.11 \mathrm{sec}$ & 92.5 & 97.5 & 90.89 & -0.46 & -0.49 & 31.5 & 17.5 & 435 \\
\hline 2.0 & $26.43 \mathrm{sec}$ & 96.0 & 104.0 & 95.03 & -0.48 & -0.52 & 33.0 & 22.0 & 425 \\
\hline 1.5 & $59.46 \mathrm{sec}$ & 99.0 & 96.0 & 90.64 & -0.48 & -0.50 & 31.5 & 22.5 & 419 \\
\hline 1.0 & $200.54 \mathrm{sec}$ & 100.0 & 98.0 & 89.46 & -0.50 & -0.49 & 32.0 & 26.0 & 406 \\
\hline \hline
\end{tabular}

for assuring a reasonable runtime and an approximate solution which is not far from the ground-truth.

In the second experiment, we decided to use $\varepsilon=1.5$ for digital annulus fitting, for all the input data sets (i.e., different numbers of outliers, $100 \mathrm{n}$ for $n=0, \ldots, 10)$. As seen in Table 2 and Figure 5] numbers of inliers are always greater than 400 (i.e., the number of inliers for the ground-truth digital annulus) and the parameter values are almost similar, while the runtimes are linear - almost constant (around 1 minute). For comparison, the exact algorithm 8 with computational complexity in $O\left(N^{3} \log N\right)$ was also applied. The obtained parameter values are $a=99.25, b=99.5$ and $r=90.65$ for all $n$. The runtimes, shown in Table 2 and Figure 6 are indeed polynomial with respect to the number of points.

Table 2. Results of digital annulus fitting with $\varepsilon=1.5$ to the data with $100 n$ outliers. The runtimes of the approximation method and the exact method are compared.

\begin{tabular}{|c|c|c|c|c|c|c|c|c|c|c|}
\hline \multirow{2}{*}{$\begin{array}{c}\text { outliers } \\
(100 n)\end{array}$} & \multirow{2}{*}{$\begin{array}{l}\text { runtime } \\
\text { (approx. } \\
\text { method) }\end{array}$} & \multicolumn{8}{|c|}{ parameters } & \multirow{2}{*}{$\begin{array}{c}\text { runtime } \\
\text { (exact. } \\
\text { method) }\end{array}$} \\
\hline & & $a$ & $\bar{b}$ & $r$ & $A$ & $B$ & $\bar{C}$ & $C^{\prime}$ & inliers & \\
\hline 0 & $46.20 \mathrm{sec}$ & 96.0 & 99.0 & 90.65 & -0.48 & -0.495 & 31.5 & 22.5 & 400 & $1 \mathrm{~m} 22 \mathrm{sec}$ \\
\hline 100 & $59.46 \mathrm{sec}$ & 96.0 & 99.0 & 90.65 & -0.48 & -0.495 & 31.5 & 22.5 & 419 & $2 \mathrm{~m} 45 \mathrm{sec}$ \\
\hline 200 & $61.48 \mathrm{sec}$ & 94.5 & 96.0 & $\overline{92.46}$ & -0.47 & -0.48 & 28.5 & 19.5 & 437 & $4 \mathrm{~m} 45 \mathrm{sec}$ \\
\hline 300 & $63.83 \mathrm{sec}$ & 94.5 & 96.0 & 92.45 & $\mid-0.47$ & -0.48 & 28.5 & 19.5 & 454 & $7 \mathrm{~m} 43 \mathrm{sec}$ \\
\hline 400 & $63.94 \mathrm{sec}$ & 94.5 & 96.0 & 92.45 & \begin{tabular}{|c|}
-0.47 \\
\end{tabular} & -0.48 & 28.5 & 19.5 & 476 & $11 \mathrm{~m} 37 \mathrm{sec}$ \\
\hline 500 & $64.57 \mathrm{sec}$ & 94.5 & 96.0 & 92.45 & -0.47 & -0.48 & 28.5 & 19.5 & 500 & $16 \mathrm{~m} 43 \mathrm{sec}$ \\
\hline 600 & $65.00 \mathrm{sec}$ & 96.0 & 99.0 & 90.65 & -0.48 & -0.495 & 31.5 & 22.5 & 519 & $23 \mathrm{~m} 8 \mathrm{sec}$ \\
\hline 700 & $65.22 \mathrm{sec}$ & 96.0 & 102.0 & 90.66 & -0.48 & -0.51 & 33.0 & 24.0 & 551 & $30 \mathrm{~m} 49 \mathrm{sec}$ \\
\hline 800 & $65.77 \mathrm{sec}$ & $\overline{96.0}$ & 99.0 & 90.65 & -0.48 & -0.495 & 31.5 & 22.5 & 570 & $40 \mathrm{~m} 24 \mathrm{sec}$ \\
\hline 900 & $66.04 \mathrm{sec}$ & 96.0 & 99.0 & 90.65 & -0.48 & -0.495 & 31.5 & 22.5 & 591 & $51 \mathrm{~m} 3 \mathrm{sec}$ \\
\hline 1000 & $66.18 \mathrm{sec}$ & 96.0 & 99.0 & 90.65 & -0.48 & -0.495 & 31.5 & 22.5 & 618 & $64 \mathrm{~m} 8 \mathrm{sec}$ \\
\hline \multicolumn{2}{|c|}{ ground truth } & 100.0 & 100.0 & \begin{tabular}{|c|}
90.5 \\
\end{tabular} & -0.50 & -0.50 & 30.20 & 28.84 & 400 & \\
\hline
\end{tabular}




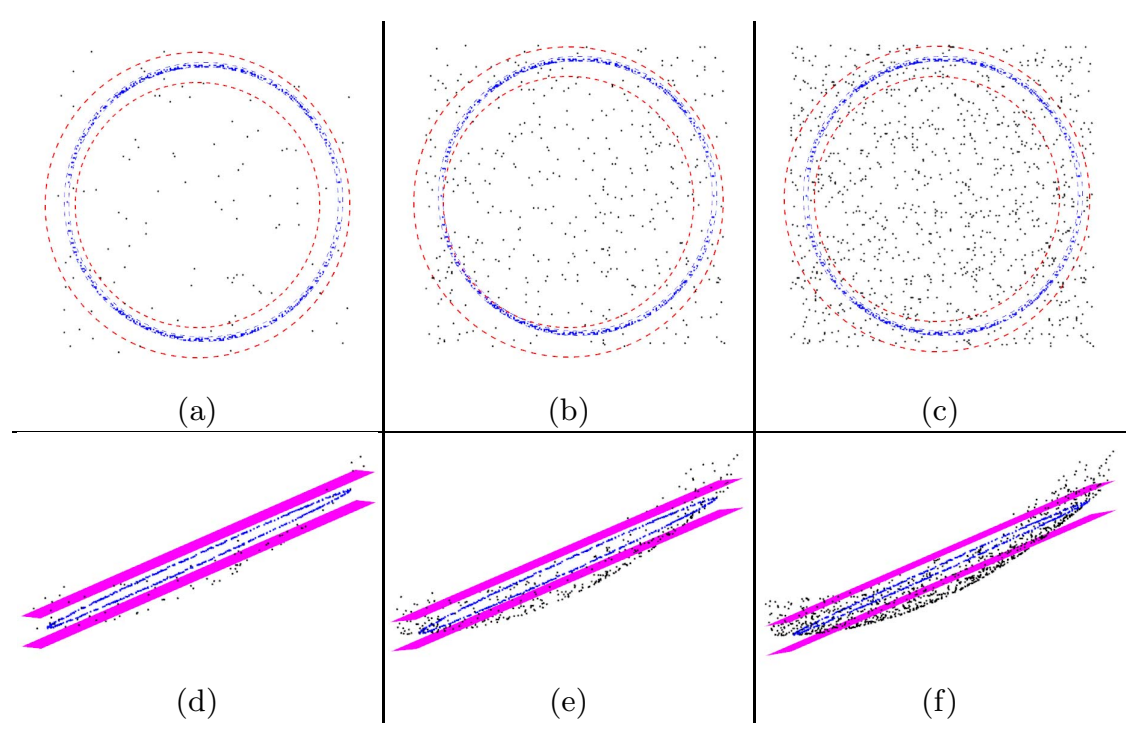

Fig. 5. The fitted digital annuli (colored in red) with $100 n$ outliers in input for $n=1$ (a), $n=4$ (b), $n=10$ (c). They contains the true digital annulus (colored in blue). The converted digital planes are also illustrated for $n=1$ (d), $n=4$ (e), $n=10$ (f).

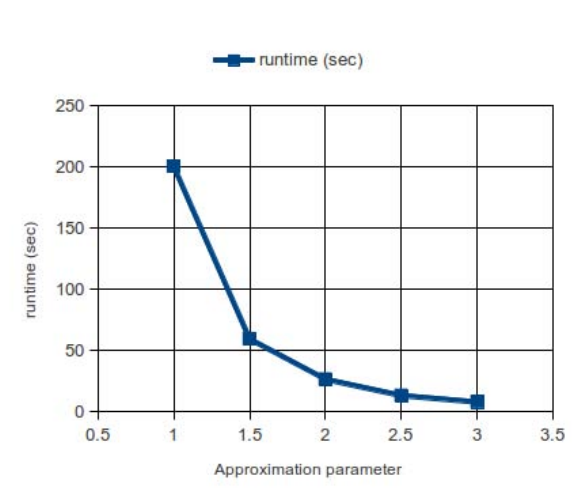

(a)

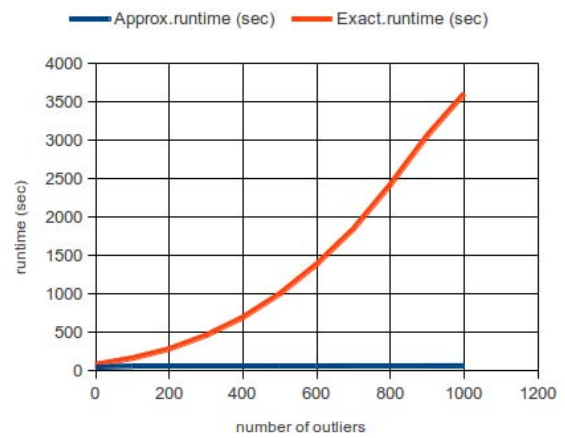

(b)

Fig. 6. Runtimes of digital annulus fitting with respect to varied $\varepsilon$ (a) and to number of outliers (b)

\section{Conclusion}

This paper presented an approximation method for fitting a digital annulus. Given $N$ points in a $[0, \delta]^{2}$ grid, a width $w$ and an approximation parameter $\varepsilon$, the fitted digital annulus that contains the maximum number of points with a 
width $w+5 \frac{\delta}{r} \varepsilon$, is found in $O\left(N+\varepsilon^{-3} \log ^{O(1)}\left(\varepsilon^{-1}\right)\right)$ time. The method is linear in the number of points $N$, but it is polynomial in approximation parameter $\varepsilon$. Therefore, we need to find an appropriate $\varepsilon$ for assuring a reasonable runtime. The method is also robust to outliers. The number of inliers converges to the optimal solution when $\varepsilon$ decreases.

\section{References}

1. Boyd, S., Vandenberghe, L.: Convex optimization. Cambridge University Press (2004)

2. Press, W.H., Teukolsky, S.A., Vetterling, W.T., Flannery, B.P.: Numerical recipes: the art of scientific computing, 3rd edn. Cambridge University Press (2007)

3. Rousseeuw, P.J.: Least median of squares regression. Journal of the American Statistical Association 79(388), 871-880 (1984)

4. Fischler, M.A., Bolles, R.C.: Random sample consensus: a paradigm for model fitting with applications to image analysis and automated cartography. Communications of the ACM 24(6), 381-395 (1981)

5. Zrour, R., Kenmochi, Y., Talbot, H., Buzer, L., Hamam, Y., Shimizu, I., Sugimoto, A.: Optimal consensus set for digital line and plane fitting. International Journal of Imaging Systems and Technology 21, 45-47 (2011)

6. Aiger, D., Kenmochi, Y., Talbot, H., Buzer, L.: Efficient Robust Digital Hyperplane Fitting with Bounded Error. In: Debled-Rennesson, I., Domenjoud, E., Kerautret, B., Even, P. (eds.) DGCI 2011. LNCS, vol. 6607, pp. 223-234. Springer, Heidelberg (2011)

7. Andres, E., Jacob, M.-A.: The Discrete Analytical Hyperspheres. IEEE TVCG 3(1), 75-86 (1997)

8. Largeteau-Skapin, G., Zrour, R., Andres, E.: $O\left(n^{3} \log n\right)$ Time Complexity for the Optimal Consensus Set Computation for 4-Connected Digital Circles. In: GonzalezDiaz, R., Jimenez, M.-J., Medrano, B. (eds.) DGCI 2013. LNCS, vol. 7749, pp. 241-252. Springer, Heidelberg (2013)

9. da Fonseca, G.D., Mount, D.M.: Approximate range searching: The absolute model. Comput. Geom. 43, 434-444 (2010)

10. O'Rourke, J., Kosaraju, S.R., Megiddo, N.: Computing circular separability. Discrete and Computational Geometry 1(1), 105-113 (1986)

11. Andres, E., Acharya, R., Sibata, C.: Discrete Analytical Hyperplanes. GMIP 59(5), 302-309 (1997)

12. Provot, L., Gérard, Y.: Estimation of the Derivatives of a Digital Function with a Convergent Bounded Error. In: Debled-Rennesson, I., Domenjoud, E., Kerautret, B., Even, P. (eds.) DGCI 2011. LNCS, vol. 6607, pp. 284-295. Springer, Heidelberg (2011)

13. Fiorio, C., Toutant, J.-L.: Arithmetic Discrete Hyperspheres and Separatingness. In: Kuba, A., Nyúl, L.G., Palágyi, K. (eds.) DGCI 2006. LNCS, vol. 4245, pp. 425-436. Springer, Heidelberg (2006)

14. Costa, L.F.D., Cesar, R.M.: Shape analysis and classification: Theory and practice, 1st edition. CRC Press (2000)

15. Ding, D., Ping, X., Hu, M., Wang, D.: Range image segmentation based on randomized Hough transform. Pattern Recognition Letters 26(13), 2033-2041 (2005) 